\title{
Efectos de una intervención educativa y de participación comunitaria en el control de la malaria en Buenaventura, Colombia
}

\author{
Beatriz Eugenia Alvarado', Alberto Alzate ${ }^{2}$, Julio Cesar Mateus ${ }^{3,4}$, Rocío Carvajal ${ }^{3}$ \\ ${ }^{1}$ Grupo AntroPacífico, Unidad de Epidemiología Clínica, Universidad del Cauca, Popayán, Colombia. \\ ${ }^{2}$ Fundación Carvajal, Cali, Colombia. \\ ${ }^{3}$ División Salud, Fundación FES, Cali, Colombia. \\ ${ }^{4}$ Escuela de Salud Pública, Universidad del Valle, Cali, Colombia.
}

Introducción. Una intervención para el control de la malaria no aleatoria compuesta por cuatro componentes y basada en atención primaria fue implementada en Buenaventura, Colombia. Objetivo. Evaluar el efecto de la intervención en conocimientos y prácticas en malaria, atención de eventos febriles y frecuencia de malaria en tres comunas de Buenaventura.

Materiales y métodos. Se realizó una evaluación post-intervención con grupo control no equivalente. Se identificaron dos grupos de no contacto de más y menos seis meses de residencia en la zona, respectivamente, y un grupo contacto con la intervención. Se entrevistaron 661 mujeres jefes de hogar. El contacto fue el haber estado expuesto al menos a uno de los cuatro componentes de la intervención.

Resultados. El 14\% de los entrevistados tuvieron contacto con la intervención. El evento febril fue mejor atendido en el grupo de personas contacto que en el grupo "sin contacto" que habían vivido en el lugar por más de seis meses. Los sin contacto con menos de seis meses en el lugar reportaron menor uso de toldillos (OR: 0,46; IC 95\%: 0,23-0,93) y de práctica de fumigación (OR: 0,38; IC 95\%: 0,19-0,75). El análisis de tendencia de casos de malaria mostró disminución de $25 \%$ a $17 \%$ de casos aportados por los grupos intervenidos, seis años después de la intervención.

Conclusión. La estrategia educativa es efectiva para elevar los conocimientos y modificar las prácticas de la población urbana de Buenaventura frente a la malaria.

Palabras clave: atención primaria; malaria/prevención \& control; conocimientos, actitudes y práctica en salud; estudios de intervención; evaluación de programas y proyectos de salud, Colombia.

Effects of an educational and participatory community intervention on malaria control in Buenaventura, Colombia

Introduction. A four-component, non randomized, primary-care based intervention for malaria control was implemented in Buenaventura, Colombia.

Objective. To evaluate the effect of the applied intervention on knowledge about malaria, attention of febrile events and frequency of malaria occurrence in three communities of Buenaventura.

Materials and methods. A post-intervention evaluation with a non-equivalent control group was performed. Two non-intervened groups (those residing more and less than six months, respectively, in the area) and one intervened group were identified. We interviewed 661 women household heads. Contact was defined as having been exposed to at least one of the four intervention components.

Results. Fourteen percent of the respondents had contact with the intervention. The attention of a febrile episode was better in those who had contact with the intervention than in the nonintervened ones who had resided in the area for more than six months. Those without contact and with less than six months stay in the area reported lower use of bed-nets (OR:0.46; 95\% $\mathrm{Cl}: 0.23-0.93)$ and less fumigation practices (OR:0.38; 95\% Cl:0.19-0.75). The analysis of the 
malaria case trend showed a reduction in the proportion of cases contributed by the intervened communities, from $25 \%$ to $17 \%$, six years after the intervention.

Conclusion. An educational strategy is effective to enhance knowledge and modify the practices of the urban population of Buenaventura with respect to malaria.

Key words: primary care; malaria/prevention \& control; health knowledge, attitudes, practice, intervention studies; program evaluation; Colombia.

Teniendo en cuenta el carácter local de la lucha contra la malaria y el papel del hombre en la perpetuación de esta enfermedad, la Organización Mundial de la Salud (OMS) sugirió la implementación de los programas antimalaricos en el marco de los principios de atención primaria (1). El control de la malaria bajo los supuestos de la atención primaria se enfoca en el mantenimiento de las medidas de control, en el acceso oportuno al diagnóstico y el tratamiento, en el reforzamiento de la capacidad local en materia de investigación básica y aplicada, y en la evaluación regular de actividades contra el paludismo (2). El entrenamiento de personas de la comunidad en el diagnóstico y tratamiento oportuno de los enfermos con paludismo (3), la educación para el reconocimiento, diagnóstico y tratamiento de personas febriles (4), la impregnación con permetrina de toldillos (5) y otras estrategias integrales han logrado la disminución de la prevalencia de malaria (3) y de la mortalidad y morbilidad infantil por esta causa (4).

La puesta en marcha y continuidad de las medidas para el control de la malaria en Colombia bajo los supuestos de la atención primaria ha sido lenta y presenta muchas debilidades, especialmente después de la nueva reforma en salud (6). De allí que varios grupos de investigación en Colombia desarrollaran iniciativas para involucrar a las comunidades y fortalecer el papel de los programas de control municipales. La creación de cooperativas de venta e impregnación de toldillos en la costa Pacífica chocoana (7), la educación en malaria de las comunidades, el fortalecimiento de la red de diagnóstico y el control del vector con apoyo intersectorial fueron algunas de estas

\footnotetext{
Correspondencia:

Rocío Carvajal Barona, calle 23 norte No. 5 AN 47, Cali, Colombia. Tel. (2) 6534141.

rocaba70@fundacionfes.org
}

Recibido: 09/02/06; aceptado: 25/07/06 iniciativas (8). Los resultados de esta última experiencia, por ejemplo, muestran un impacto en la reducción de la prevalencia y la gravedad de la malaria, un incremento en el uso de medidas de prevención y una reducción de la mortalidad por paludismo.

Al igual que en otras zonas endémicas, el control de la malaria en Buenaventura ha recaído en el programa de enfermedades tropicales del municipio, que se encarga del rociamiento extra e intradomiciliario, del mantenimiento de la red de diagnóstico y tratamiento de la malaria en los puestos de salud y, ocasionalmente, de la impregnación de toldillos con permetrina. Sin embargo, los datos de malaria mostraban desde 1991 un aumento de casos en el área urbana, especialmente en las comunas 10,11 y 12 .

Por ello, y frente a la posibilidad de fortalecer el programa de control de la malaria, se decidió diseñar una estrategia fundamentada en los principios básicos de la atención primaria en salud (Carvajal R, Mosquera J, Carrasquilla G. El mundo de la malaria: aprendamos a manejarlo en comunidad. Una experiencia educativa en salud en la costa Pacífica colombiana. Observaciones no publicadas) (9). Esta intervención fue diseñada por el Instituto de Salud del Pacífico (INSALPA) y la Fundación FES para fortalecer el componente de la participación comunitaria en el control de la malaria mediante la aplicación de cuatro tipos de intervenciones: 1) entrenamiento de personas de la comunidad en el uso del material educativo (Carvajal R, Mosquera J, Carrasquilla G. El mundo de la malaria: aprendamos a manejarlo en comunidad. Una experiencia educativa en salud en la costa Pacífica colombiana. Observaciones no publicadas);2) detección y control de criaderos de anofeles; 3) generación de programas de prevención de la malaria tales como la producción y venta de toldillos, y 4) adiestramiento de personas para el diagnóstico microscópico y el 
tratamiento de la enfermedad en los barrios. Los objetivos de la intervención fueron transmitir o aumentar los conocimientos sobre la malaria y sus formas de prevención; establecer grupos encargados de la identificación y control de los criaderos de anofeles, y aumentar el uso de servicios de diagnóstico comunitario.

El presente documento evalúa la cobertura y los efectos de dicha intervención entre 1995 y 1997 en términos de los conocimientos y prácticas (en prevención y atención), la frecuencia de la malaria entre los pobladores de los barrios intervenidos y la frecuencia de la malaria en el total de personas de las comunas 10, 11 y 12 de Buenaventura.

\section{Materiales y métodos}

\section{Tipo de estudio}

Se realizó un estudio cuasi-experimental posterior a la prueba sin grupo de control equivalente (10) para evaluar los efectos de la intervención tres años después de aplicada en la comunas 10,11 y 12 de Buenaventura. Estas comunas se caracterizan por ser sitios de expansión de la ciudad y comparten condiciones socioeconómicas similares y cifras de alta prevalencia de malaria, especialmente la comuna $12(9,11)$.

El diseño se basó en la comparación de los conocimientos, prácticas y frecuencia de la malaria entre quienes habían estado expuestos a la intervención y una muestra de personas no expuestas a la intervención. Los expuestos y no expuestos fueron identificados por medio de una encuesta en los barrios intervenidos.

\section{Descripción de la intervención}

En 1995, los integrantes de grupos comunitarios de las comunas 10, 11 y 12 de Buenaventura, que habían participado en la creación de un material educativo denominado El mundo de la malaria: aprendamos a manejarlo en comunidad, fueron capacitados para usar el material educativo y multiplicar la experiencia en sus barrios (Carvajal R, Mosquera J, Carrasquilla G. El mundo de la malaria: aprendamos a manejarlo en comunidad. Una experiencia educativa en salud en la costa Pacifica colombiana. Observaciones no publicadas). Los talleres de capacitación tuvieron una duración de ocho días en jornadas diarias de ocho horas. En esos primeros talleres participaron aproximadamente 78 personas. Los participantes eran trabajadores de la salud o promotores de salud comunitarios (personas con escolaridad mínima de básica primaria que reciben un año de capacitación en cuidados básicos de salud y remuneración por su labor), voluntarios de salud (personas de la comunidad que no han recibido capacitación formal, pero que conocen sobre cuidados de salud y no reciben remuneración por su trabajo) y maestros o educadores (personas con formación mínima de bachilleres, quienes posteriormente hacen una licenciatura en educación).

Una vez capacitados, estos facilitadores y el equipo del INSALPA procedieron a multiplicar la experiencia en sus comunidades de origen. En este proceso se crearon grupos para el manejo de criaderos y para generar ingresos destinados a microempresas de toldillos impregnados con insecticida. Algunos facilitadores fueron además entrenados para hacer el diagnóstico de malaria en sus comunidades. El seguimiento fue de dos años a partir de la capacitación, con valoraciones en el transcurso de la intervención comunitaria y una evaluación de todo el proceso al final del tercer año.

\section{Selección de la muestra}

El área cubierta por la investigación incluyó las comunas 10, 11 y 12 de la zona urbana de Buenaventura. La comuna 12 fue intervenida con la estrategia en la totalidad de sus barrios, mientras que en las comunas 11 y 10 se intervino un solo barrio en cada una. La unidad de muestreo escogida fueron las manzanas, debido a la dificultad para la realización de muestreos más complejos en estos barrios de asentamientos no planificados donde, por lo general, no existen direcciones. La unidad de análisis planteada fueron las mujeres jefes de hogar de los barrios de las comunas intervenidas con la estrategia educativa.

La selección de los hogares a encuestar se realizó mediante la identificación en el mapa catastral de Buenaventura de las manzanas donde residían quienes habían recibido la capacitación 
(facilitadores) y el maletín "Mundo de la malaria". Se encontraron 24 manzanas con personas capacitadas en la comuna 12, una manzana en la comuna 11 y una manzana en la comuna 10. Las manzanas donde habitaban estas personas se denominaron "manzanas con maletín".

Bajo el supuesto de que en las manzanas donde no habitaban personas capacitadas se encontraría un número menor de personas en contacto con la intervención, se procedió a hacer una selección al azar de manzanas de las comunas 10, 11 y 12 donde no vivía ningún facilitador. A estas manzanas se las denominó "manzanas sin maletín". Para su selección se tomó la numeración dada por el mapa catastral y se seleccionó aleatoriamente una cantidad igual a la de las manzanas con maletín: 24 en la comuna 12, una en la 11 y una en la 10. Sin embargo, al aplicar la encuesta, una de las manzanas considerada como "con maletín" resultó ser una manzana "sin maletín", quedando así la muestra compuesta por 25 y 27 manzanas, respectivamente.

El número de hogares a entrevistar se calculó para estimar la proporción de personas en contacto con la intervención mediante un muestreo aleatorio simple (12). Los datos para el cálculo fueron el total de la población de las comunas bajo estudio (68.716 personas); el número de hogares de las comunas (12.394), y la proporción para estimar $10 \%$ de contacto con la estrategia y precisión de $5 \%$. El tamaño de la muestra para un nivel de confianza de $95 \%$ fue de 549 hogares. El número de hogares (mujeres jefes de hogar) encuestados fue de 661 .

En cada manzana seleccionada se invitó a participar a todas las viviendas que la componían. En las viviendas se entrevistó a las mujeres cabeza de hogar, grupo éste que constituía una de las poblaciones objeto de la estrategia (Carvajal R, Mosquera J, Carrasquilla G. El mundo de la malaria: aprendamos a manejarlo en comunidad. Una experiencia educativa en salud en la costa Pacifica colombiana. Observaciones no publicadas).

\section{Recolección de los datos}

Las entrevistas fueron realizadas por seis mujeres de Buenaventura con bachillerato completo, entrenadas previamente por los investigadores. Durante un periodo de tres meses se desplazaron a cada una de las comunas y aplicaron una encuesta semiestructurada ajustada a partir de un cuestionario utilizado en estudios anteriores en la zona (13). El cuestionario reunía información concerniente a las características demográficas del jefe del hogar, el estado socioeconómico de la familia, datos sobre conocimientos y prácticas en malaria, frecuencia de malaria en el hogar y los datos que permitían identificar si se había tenido contacto o no con la intervención.

\section{Intervención o exposición}

El contacto con la intervención se identificó como la variable exposición. Mediante 12 preguntas estructuradas se determinó si la persona entrevistada había estado o no en contacto. Una persona se clasificaba como expuesta si había participado en al menos una de las siguientes actividades: 1) talleres educativos sobre el material del maletín "Mundo de la malaria"; 2) grupos comunitarios de manejo de criaderos; 3 ) grupos comunitarios para impregnar o vender toldillos, y 4) búsqueda de atención en la red de diagnóstico de malaria del barrio. Para la definición de la exposición no se tuvo en cuenta ni la duración de los talleres, ni la calidad de la educación, ni la regularidad del contacto con la intervención.

\section{Efectos esperados}

Cobertura. Cantidad de jefes de hogar que recibió la capacitación por parte del facilitador del INSALPA, participó en grupos comunitarios o buscó atención en la red de diagnóstico.

Conocimientos sobre malaria. Los conocimientos sobre los síntomas, modo de transmisión, tratamiento y modo de prevención se averiguaban por medio de preguntas abiertas, analizadas después como variables dicotómicas (sí/no conoce).

Prácticas de prevención. Se midieron las prácticas relacionadas con la atención y la prevención del paludismo. Las prácticas de prevención sobre las que se preguntó fueron el uso reportado de toldillo (al menos una persona en casa lo usa), la impregnación de toldillos (sí/ no), el permiso dado para fumigar (sí/no) y la 
práctica de fumigación del hogar por parte de los propios habitantes (sí/no). Las prácticas de atención de la malaria se identificaron a partir de la pregunta hipotética de un caso febril en el hogar o la práctica frente al último episodio de malaria de alguna persona de la familia. En este caso se determinó si la persona se automedicaba con antipalúdicos y el lugar de inicio del tratamiento (casa/puesto de salud).

Frecuencia de malaria en casa. Se definió como el reporte de malaria (diagnosticada por gota gruesa) en los 15 días anteriores en alguna persona de la casa.

Frecuencia de malaria en los barrios. Se buscó información sobre la tendencia de la enfermedad en los barrios de estas comunas desde 1991 hasta 1997 y se relacionó dicho comportamiento con las actividades realizadas en la intervención y las estrategias de prevención del programa de enfermedades tropicales del municipio.

\section{Variables de confusión}

Debido a que la intervención no fue asignada al azar, los grupos expuestos y no expuestos pudieron diferir en algunos factores que influirían en el reporte de las prácticas y la frecuencia de la malaria. Los expuestos y los no expuestos a la intervención fueron comparados en términos de edad, educación del jefe del hogar y estado socioeconómico, así como por el número de habitantes de la casa. Para medir el estado socioeconómico se preguntó por el número de electrodomésticos en la vivienda (nevera, estufa y televisor), el tipo de vivienda definida a partir de la clase de suelo y paredes, y la propiedad de la vivienda.

\section{Análisis}

Los análisis iniciales mostraron que el contacto con la intervención entre aquellos que tenían menos de seis meses de estar en el barrio fue de $0 \%$ (figura 1). Se definieron entonces dos tipos de personas sin contacto con la intervención o no expuestos: aquellos que llevaban menos de 6 meses viviendo en el barrio, a quienes se les denominó en adelante "seguramente no contacto" $(n=74)$, y aquellos con más de seis meses en el barrio o "posiblemente no contacto" $(n=494)$.

El análisis del efecto se realizó comparando los conocimientos, las prácticas preventivas, las prácticas de atención de la enfermedad y la frecuencia de malaria de los expuestos y de los dos grupos de no expuestos. En una intervención se pueden medir los siguientes efectos: el efecto directo, o sea la diferencia en conocimientos,

Figura 1. Probabilidad de contacto con la intervención (1).

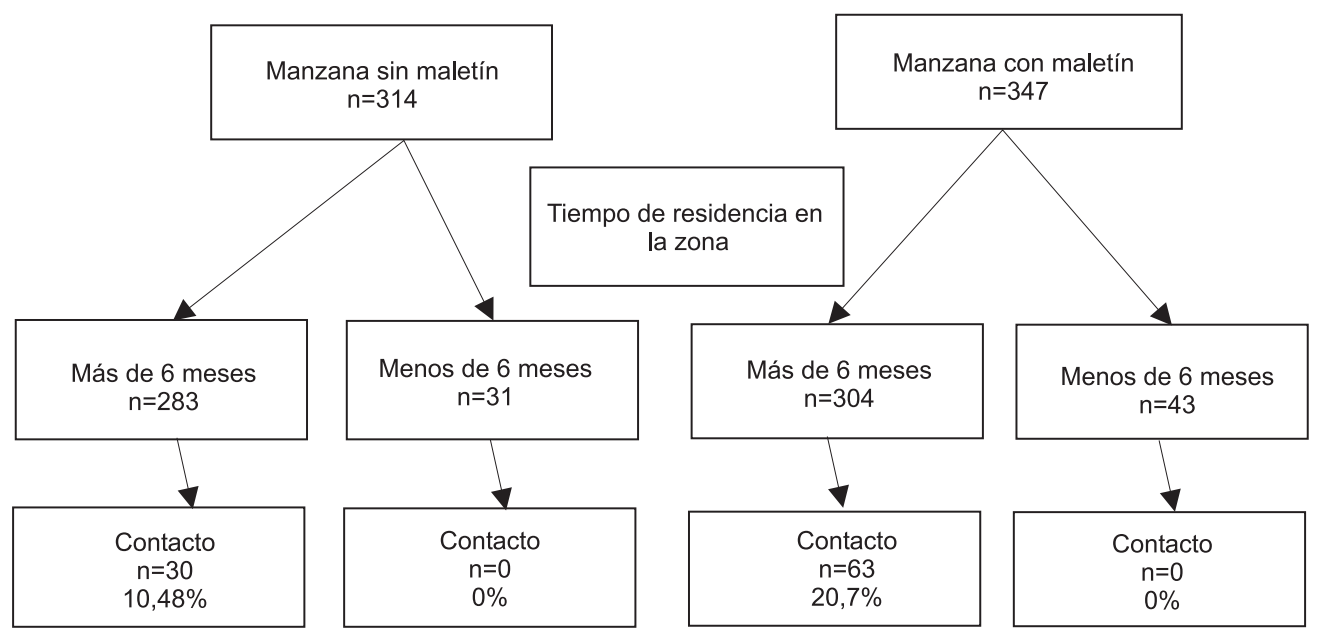

(1) La posibilidad de contacto con la intervención se considera asociada a la presencia del maletín con el programa educativo. 
prácticas y frecuencia de malaria entre quienes tuvieron contacto con la intervención y quienes no lo tuvieron, pero que pudieron haberlo tenido (contacto - posiblemente sin contacto); el efecto indirecto, es decir la diferencia entre posiblemente sin contacto y seguramente no contacto, que mide la "contaminación", pues el hecho de vivir en un barrio intervenido expone a las personas de manera no formal a la intervención, $y$, por último, el efecto total, o sea la diferencia entre los contactos y los seguramente no contactos. Este efecto no presenta contaminación, ya que el grupo de menos de seis meses de residencia en el barrio no tuvo ninguna posibilidad de exponerse a la intervención.

Aunque estos tres tipos de efectos se han definido para la evaluación de vacunas y no para evaluar programas educativos, pensamos que el supuesto es el mismo: al igual que las enfermedades infeccionas, los conocimientos y las prácticas se pueden transmitir (14).

Mediante análisis bivariados se buscaron diferencias de proporciones con niveles de significación del $95 \%$. Se utilizaron regresiones logísticas múltiples para evaluar el efecto de la intervención en cuanto a conocimientos, prácticas y frecuencia de la enfermedad. En los modelos se incluyeron las variables sociodemográficas del jefe del hogar (edad y escolaridad en años de estudio completados) y su nivel socioeconómico (número de electrodomésticos en casa, tipo de suelo y propiedad de la vivienda); además, en los modelos para las prácticas se incluyeron los conocimientos.

De igual manera, para medir el efecto del programa sobre la enfermedad se buscó la tendencia de la enfermedad en los barrios de estas comunas desde 1991 hasta 1997, y se relacionó dicho comportamiento con las actividades realizadas en la intervención y los programas de prevención del programa de enfermedades tropicales del municipio. Los datos fueron procesados en los programas Epi-Info 6.04 (12) y STATA (15).

\section{Resultados}

Se visitaron y se entrevistaron 661 mujeres jefes de hogar; de ellas, 347 residían en 25 manzanas con el maletín "Mundo de la malaria" y 314 en 27 manzanas sin maletín.
El 93\% de las personas entrevistadas vivía en la comuna 12. El promedio y la desviación estándar de la edad de las jefes de hogar encuestadas fue de 35,5 \pm 12 años y el promedio de años de estudio fue de $5 \pm 3,3$ años. Estas personas habían vivido en el barrio en promedio $81 \pm 71,4$ meses. El $77 \%$ de las jefes de hogar vivía en casa propia, con predominio de pisos de cemento y baldosa $(72,6 \%)$. El promedio de habitaciones por casa fue de 1,89 y el de personas por casa, 5,0 . Cincuenta y cuatro por ciento de los hogares contaba con los tres electrodomésticos contemplados (televisor, nevera y estufa), mientras que $8 \%$ no contaba con ninguno de ellos.

\section{Comparación de los intervenidos y no intervenidos}

La frecuencia de la etnia negra fue similar en los tres grupos $(p=0,08): 70 \%$ en contacto, $60,7 \%$ en posiblemente no contacto y $45,9 \%$ en seguramente no contacto.

La edad (promedio $\pm \mathrm{DE}$ ) del grupo "en contacto con la intervención" $(36,2 \pm 11,0)$ fue similar a la del grupo posiblemente no contacto" $(36,0 \pm 12,0)$, pero significativamente mayor $(p<0,05)$ a la del grupo "seguramente no contacto" $(31,1 \pm 10,0)$. También hubo una diferencia significativa $(p<$ 0,05 ) en cuanto a la escolaridad (años): "en contacto", 5,8 $\pm 3,6$; "posiblemente no contacto", $4,9 \pm 3,2$, y "seguramente no contacto", $5,2 \pm 3,3$. De igual manera, difirieron $(p<0,05)$ en lo concerniente al número de personas en la vivienda: "en contacto", 6,49 $\pm 3,4$; "posiblemente no contacto", $5,39 \pm 2,3$, y "seguramente no contacto", 4,7 $\pm 3,7$.

La posesión de la vivienda de residencia también fue estadísticamente diferente entre los grupos ( $p<0,01)$ : 92,2\% "en contacto", $80,2 \%$ en "posiblemente no contacto" y $36 \%$ en "seguramente no contacto". La posesión de tres electrodomésticos en casa no marcó diferencia entre los grupos ( $p=0,40)$ : "en contacto", $64,5 \%$; "posiblemente no contacto", 53,3\% y "seguramente no contacto", 47,3\%.

En resumen, las personas en contacto con la intervención tuvieron mayor escolaridad y mejor nivel socioeconómico que las personas del grupo "posiblemente no contacto". Sin embargo, 
comparadas con las personas "seguramente sin contacto", su escolaridad fue menor, pero su estado socioeconómico mejor.

\section{Cobertura}

El 14\% (93/661) de las personas entrevistadas había estado en contacto con la intervención en cualquiera de las tres actividades definidas (figura 1). De las 93 personas que tuvieron contacto, $72 \%$ había recibido educación en malaria por medio del maletín del "Mundo de la malaria" y 54\% tuvo contacto con grupos de participación comunitaria para prevención de la malaria (criaderos y toldillos). En los dos barrios en los que el programa adecuó un microscopio para el diagnóstico, $23 \%$ de los encuestados consultó en caso de fiebre al microscopista comunitario. La encuesta mostró que, ante un episodio febril, los encuestados prefirieron los servicios formales, como el puesto de salud o el programa de enfermedades tropicales. El 37,3\% de las actividades educativas y el $55 \%$ de las actividades de participación comunitaria se había realizado en el año anterior.

Como se comentó anteriormente, la cobertura estuvo relacionada con la presencia de maletín en la manzana y con el tiempo de residencia en el barrio (figura 1). En las manzanas con maletín, el contacto de las personas con la intervención fue significativamente mayor $(p=0,001 ; 18,1 \%$ IC $95 \%$ : 14,1 a 22,4$)$ que en las manzanas sin maletín (9,5\% IC 95\%: 6,6 a 13,2).

\section{Efectos directos, indirectos y totales}

El cuadro 1 presenta la estimación de los efectos directos, indirectos y totales en síntomas, prácticas y frecuencia de la malaria, sin ajustar por las diferencias entre los grupos de exposición. No hubo diferencias en el conocimiento del zancudo y $99 \%$ de los entrevistados sabía que así se transmitía la enfermedad. Las personas en contacto con la estrategia conocían más de los tratamientos antimaláricos, de los síntomas, de la forma en que se cría el zancudo y del manejo de criaderos como medida de prevención que las de los dos grupos de "no contacto" (efecto directo $y$ total significativos) (cuadro1).

El uso de medidas de prevención no fue mayor en quienes tuvieron contacto que en los "posiblemente no contacto" (ausencia de efecto directo), pero sí mayor que en los "seguramente no contacto" (efecto total en uso de toldillo, fumigación de la casa y permiso para rociamiento).

Las prácticas de atención a una persona febril fueron más adecuadas entre los "contacto" que entre los "posiblemente no contacto" (efecto directo), pero no hubo diferencias significativas con los "seguramente no contacto" (ausencia de efecto total). El efecto indirecto se observó en el conocimiento de tratamientos y en las prácticas de fumigación. Sin embargo, los "posiblemente no contacto" se automedicaban más que los "seguramente no contacto". La frecuencia de los reportes de malaria en los tres grupos fue muy baja y no se encontraron diferencias significativas.

\section{Análisis multivariados}

Las asociaciones significativas encontradas en el análisis anterior se examinaron en modelos de regresión logística ajustados según las variables de confusión (educación, edad, nivel socioeconómico), y en el ámbito de las prácticas, según los conocimientos (en el caso de prácticas de prevención: uso de toldillo, conocimientos sobre prevención, conocimiento sobre toldillos; en el caso de prácticas de atención: conocimiento de antipalúdicos).

Comparados con los "contacto", los "seguramente no contacto" tuvieron menos probabilidad de conocer sobre los criaderos, los tratamientos y las medidas de prevención (efecto total), pero con respecto a los "posiblemente no contacto", la diferencia no fue significativa para ninguno de los conocimientos.

En cuanto a las prácticas, la intervención produjo un efecto significativo en el uso del toldillo, la fumigación, el permiso para rociar y en la búsqueda de atención ante una sospecha de malaria cuando se compararon los intervenidos con los "seguramente no contacto" (cuadro 2), pero la intervención no tuvo efecto en las prácticas preventivas entre los "posiblemente no contacto" y sólo mostró un efecto significativo en la búsqueda apropiada de atención.

Los "seguramente no contacto" tenían menos conocimientos de tratamientos antipalúdicos (OR: 
0,36; IC 95\%: 0,21 a 0,64); fumigaban menos (OR: 0,47; IC 95\%: 0,28 a 0,79); permitían menos el rociamiento (OR: 0,36; IC 95\%: 0,15 a 0,87), y usaban menos el toldillo (OR: 0,49; IC 95\%: 0,28 a 0,83 ) que los posiblemente no contacto (efecto indirecto).

\section{Análisis de tendencia de la malaria}

Se analizó la información recogida en Buenaventura de casos de paludismo confirmados entre 1992 y 1997. La tendencia de estos casos en el municipio fue claramente hacia su disminución desde 1994. En los barrios que fueron

Cuadro 1. Efectos directos, indirectos y totales en conocimientos, prácticas y frecuencia de la malaria'.

\begin{tabular}{|c|c|c|c|c|c|c|}
\hline Variable & $\begin{array}{l}\text { Seguramente } \\
\text { no contacto } \\
n=74\end{array}$ & $\begin{array}{c}\text { Probablemente } \\
\text { no contacto } \\
\text { n = 494 } \\
\text { B }\end{array}$ & $\begin{array}{c}\text { Contacto } \\
n=93 \\
\text { C }\end{array}$ & $\begin{array}{l}\text { Efecto } \\
\text { directo } \\
\text { C - B }\end{array}$ & $\begin{array}{c}\text { Efecto } \\
\text { indirecto } \\
\text { B - A }\end{array}$ & $\begin{array}{c}\text { Efecto } \\
\text { total } \\
\text { C - A }\end{array}$ \\
\hline \multicolumn{7}{|l|}{ Conocimientos } \\
\hline Conocimiento de síntomas (al menos 3) & 41,9 & 45,9 & 52,7 & 6,8 & 4,0 & 10,8 \\
\hline Conocimiento de criadero, sí & 83,8 & 89,7 & 95,7 & 6,0 & 5,9 & $11,9^{*}$ \\
\hline Conoce tratamiento (al menos uno) & 68,9 & 86,6 & 93,5 & 6,9 & $17,7^{*}$ & $24,6^{*}$ \\
\hline Conoce aralen, sí & 35,1 & 50,6 & 62,4 & $11,8^{*}$ & $15,5^{\star}$ & $27,3^{*}$ \\
\hline Conoce prevención (al menos dos medidas) & 59,5 & 66,8 & 75,3 & 8,5 & 7,3 & $15,8^{*}$ \\
\hline Conoce toldillo, sí & 71,0 & 73,7 & 68,9 & $-4,8$ & 2,7 & $-2,1$ \\
\hline Conoce fumigación, sí & 45,9 & 49,6 & 50,5 & 0,9 & 3,7 & 4,6 \\
\hline Conoce manejo de criadero, sí & 48,6 & 50,2 & 68,9 & $18,7^{*}$ & 1,6 & $20,3^{*}$ \\
\hline \multicolumn{7}{|l|}{ Prácticas de prevención } \\
\hline Usa toldillo, alguien en casa & 63,5 & 72,4 & 75,3 & 2,9 & 8,9 & $11,8^{*}$ \\
\hline Fumigación, sí & 50,0 & 66,6 & 74,2 & 7,6 & $16,6^{*}$ & $24,2^{*}$ \\
\hline Permite rociamiento, sí & 89,2 & 95,5 & 97,8 & 2,3 & 6,3 & $8,6^{*}$ \\
\hline \multicolumn{7}{|l|}{ Prácticas de atención } \\
\hline Inicia atención, casa & 83,2 & 86,4 & 77,8 & $-8,6^{*}$ & 3,2 & $-5,4$ \\
\hline Automedican antimalaricos, sí & 24,3 & 34,2 & 26,1 & $-8,1$ & 9,9 & 1,8 \\
\hline \multicolumn{7}{|l|}{ Frecuencia de enfermedad } \\
\hline Malaria en 15 días anteriores & 2,7 & 1,2 & 1,1 & $-0,1$ & $-1,5$ & $-1,6$ \\
\hline
\end{tabular}

1. Diferencias calculadas con la prueba de ji al cuadrado.

* $p$ menor de 0,05 .

Cuadro 2. Modelos logísticos múltiples para los conocimientos y las prácticas. Efecto total y directo.

\begin{tabular}{|c|c|c|c|c|}
\hline \multirow[t]{2}{*}{ Efectos } & \multicolumn{2}{|c|}{$\begin{array}{l}\text { Seguramente no } \\
\text { contacto vs. contacto }\end{array}$} & \multicolumn{2}{|c|}{$\begin{array}{c}\text { Posiblemente } \\
\text { no contacto vs. contacto }\end{array}$} \\
\hline & OR & IC $95 \%$ & OR & IC $95 \%$ \\
\hline \multicolumn{5}{|l|}{ Conocimientos* } \\
\hline Criaderos, sí & 0,28 & $0,08-0,94$ & 0,44 & $0,15-1,25$ \\
\hline En síntomas (al menos 3) & 0,64 & $0,34-1,19$ & 0,73 & $0,46-1,15$ \\
\hline Tratamientos, sí & 0,17 & $0,06-0,46$ & 0,48 & $0,20-1,15$ \\
\hline $\begin{array}{l}\text { En medidas de prevención (al menos } 2 \text { ) } \\
\text { Prácticas de prevención** }\end{array}$ & 0,50 & $0,25-0,97$ & 0,71 & $0,42-1,18$ \\
\hline Uso de toldillo, sí & 0,46 & $0,23-0,93$ & 0,95 & $0,56-1,62$ \\
\hline Fumigación de la casa, sí & 0,38 & $0,19-0,75$ & 0,79 & $0,47-1,32$ \\
\hline $\begin{array}{l}\text { Permite rociamiento, sí } \\
\text { Prácticas de atención }{ }^{\star *}\end{array}$ & 0,18 & $0,03-0,91$ & 0,51 & $0,11-2,22$ \\
\hline Automedicación, no & 1,07 & $0,51-2,21$ & 0,75 & $0,45-1,12$ \\
\hline Inicia atención en puesto de salud & 0,75 & $0,33-1,69$ & 0,55 & $0,31-0,96$ \\
\hline
\end{tabular}

* Modelos ajustados por educación, edad y condiciones socioeconómicas de los entrevistados.

**Modelos ajustados por educación, edad, condiciones socioeconómicas y conocimientos de los entrevistados. 
intervenidos por el INSALPA, la tendencia al descenso fue mayor que en los otros barrios. El aporte proporcional de casos de los barrios intervenidos disminuyó en $8 \%$ (25 a 17\%) en seis años. Incluso durante 1997, cuando el número de casos de malaria aumentó más allá de lo esperado, estos barrios aportaron menos casos de paludismo (cuadro 3).

\section{Discusión}

Se esperaba que, dada la elaboración del material educativo, las personas intervenidas con la estrategia adquirieran más conocimientos sobre el paludismo, tuvieran mejores prácticas de prevención (mayor uso del toldillo gracias a las cooperativas creadas), buscaran atención ante un episodio de malaria en la red situada en el barrio y que, en consecuencia, reportaran menor número de casos de malaria que aquellos no intervenidos residentes en la misma zona. Sin embargo, la evaluación mostró una baja cobertura de la intervención (apenas 14\%), con un efecto importante sólo en el cambio de las prácticas de atención a personas con un episodio febril. Los otros efectos observados deben verse a la luz de un posible efecto de contaminación de los grupos. Nuestros hallazgos también tienen implicaciones para la aplicación de esta intervención en otras comunidades, o para la aplicación de otro tipo de intervenciones en estas comunidades. Veamos algunos hallazgos e implicaciones en términos de cobertura y de efecto.

\section{Cobertura}

Una primera aproximación al número de personas educadas por los primeros capacitadores da una visión poco halagadora del grado de cobertura que la intervención alcanzó para difundir y extender las redes de personas capacitadas: se capacitaron 78 personas, y entre los entrevistados se encontraron 93 personas con contacto, lo que corresponde a 1,2 personas capacitadas por cada capacitador inicial. Igualmente, la disponibilidad (acceso y cercanía) de los elementos educativos y preventivos ofrecidos por la intervención fue mayor para los residentes de la comuna 12, lo cual puede deberse a que la intervención entrenó más personas en la comuna 12 porque allí se presentaba el mayor número de casos de malaria.
Cuadro 3. Porcentaje de casos de malaria aportados en tres años (1995 - 1997) por los barrios intervenidos versus otros barrios de Buenaventura.

\begin{tabular}{ccc}
\hline Año & $\begin{array}{c}\text { Barrios intervenidos } \\
(\%)\end{array}$ & $\begin{array}{c}\text { Barrios no intervenidos } \\
(\%)\end{array}$ \\
\hline 1992 & 25 & 75 \\
1993 & 23 & 77 \\
1994 & 22 & 78 \\
1995 & 18 & 82 \\
1996 & 10 & 90 \\
1997 & 17 & 83 \\
\hline
\end{tabular}

El contacto con la intervención dependió de sí la familia entrevistada vivía en la misma manzana del multiplicador y del tiempo de residencia de las personas en el barrio. En el primer caso, nuestra hipótesis es que la difusión de la información depende de factores como la familiaridad, el parentesco, o la vecindad con los capacitadores. El hecho de que las personas de reciente residencia en los barrios no tuvieran contacto con la intervención muestra que la continuidad de la intervención se perdió después de finalizar el seguimiento de los facilitadores hecho por el INSALPA. Los grupos focales y las entrevistas a profundidad con los facilitadores revelaron que estas personas tienen dificultades para la realización de las actividades educativas y de limpieza de criaderos, dificultades que se relacionan con la baja aceptación de la comunidad (y por ello buscan a las personas con las que tienen cierto grado de familiaridad) y las limitaciones como educadores (Gómez E, Alvarado BE, Serra M, Carvajal R, Carrasquilla G. Estrategia educativa "El mundo de la malaria: aprendamos a manejarlo en comunidad". Evaluación de Procesos. Observaciones no publicadas).

Por otro lado, la poca participación en las actividades de control de criaderos y en la utilización de toldillos demuestra el bajo grado de participación comunitaria de estos barrios. Las primeras actividades tenían un sistema de vigilancia que dependía directamente del INSALPA y del programa de enfermedades tropicales del municipio, mientras que las segundas dependían exclusivamente del interés de la comunidad. Siguiendo la clasificación de participación de Tarimo (16), las comunidades barriales analizadas 
Cuadro 4. Comparación de resultados. Encuesta de prevalencia de 1993 y estudio actual 1997. Buenaventura.

\begin{tabular}{lcc}
\hline Variable & $\begin{array}{c}\text { Encuesta } \\
\mathbf{1 9 9 3}(\%)\end{array}$ & $\begin{array}{c}\text { Encuesta } \\
\mathbf{1 9 9 7 - 1 9 9 8}(\%)\end{array}$ \\
\hline $\begin{array}{l}\text { Conocimientos } \\
\text { Conoce Aralen }\end{array}$ & 68,0 & 52,7 \\
$\begin{array}{l}\text { Conoce al menos 1 } \\
\text { síntoma de malaria }\end{array}$ & 82,0 & 96,4 \\
$\begin{array}{l}\text { Conoce zancudo } \\
\text { Conoce prevención }\end{array}$ & 85,0 & 99,5 \\
$\begin{array}{l}\text { Eliminación de criaderos } \\
\text { Toldillo }\end{array}$ & 35,4 & 53,1 \\
$\begin{array}{l}\text { Fumigación } \\
\text { Prácticas }\end{array}$ & 25,5 & 73,2 \\
Automedican & 17,2 & 49,8 \\
Usa toldillo & & \\
Fumigan & 71,0 & 33,0 \\
\hline
\end{tabular}

Fuente de datos de 1993: Méndez F. Epidemiología de la malaria en el área urbana de Buenaventura: Análisis de ocurrencia en el período 1987-1993 y factores asociados con la prevalencia de infección en la comuna 12. Cali: Universidad del Valle; 1994

son grupos sociales que reciben la información, pero no son capaces de generar o sostener actividades de prevención o de control sin asistencia técnica.

Respecto a la participación comunitaria para la destrucción de criaderos participaron menos personas (7\%), pero éstas fueron más persistentes: $55 \%$ participaron en el último año. La venta de toldillos fue impulsada inicialmente por el INSALPA y la gente participó, pero no perduró. Esta actividad perdió su continuidad al disolverse algunos grupos de generación de ingresos creados por el programa (diario de campo). La participación comunitaria en los programas de control de la malaria presenta problemas persistentes y nuestro caso no es la excepción (17). La experiencia en la región chocoana también mostró la importancia de promover de manera continua estas cooperativas de venta y de impregnación de toldillos, así como de la asesoria técnica durante periodos prolongados de tiempo como estrategias para el control y sostenibilidad de la participación comunitaria frente a la malaria (7).

La prevalencia del acceso a la red de microscopía fue de $5,7 \%$, cifra similar a la de $6,5 \%$ encontrada en el estudio de determinantes del uso de servicios en la comuna 12 en 1995 (18). Este estudio mostró que la percepción que tuvieron las personas de la red se relacionaba con el poco acceso al diagnóstico. Aumentar el acceso y la disponibilidad no garantiza su uso en estas poblaciones; para aumentar el acceso sería importante trabajar sobre las percepciones que las comunidades tienen de las redes barriales con el fin de garantizar una mayor utilización.

\section{Efecto de la intervención}

Al comparar a las personas intervenidas con las"posiblemente no contacto" y las "seguramente no contacto" (efectos directo y total), la intervención afectó principalmente las prácticas de cuidado ante un episodio febril o de malaria (búsqueda temprana de atención en puesto de salud). La ausencia de efecto total en la automedicación puede explicarse porque entre los "seguramente no contacto" la automedicación es muy baja debido a su falta de conocimiento de los medicamentos antipalúdicos. Otros estudios han puesto en evidencia este efecto de la educación de los pobladores en las prácticas de uso adecuado de medicamentos antimaláricos y en los comportamientos de búsqueda de atención positivos, y se ha observado que estos cambios se reflejan en la disminución de la morbilidad asociada a la malaria (19). Dada la alta prevalencia de resistencia a antimaláricos en las poblaciones del Pacífico colombiano (20), el efecto de la intervención tiene repercusiones importantes para el futuro control de la malaria.

La evaluación mostró la ausencia de efectos directos en conocimientos y prácticas de prevención. Por el contrario, lo que resalta la evaluación es que muchos de los conocimientos y prácticas en estas comunidades han resultado en un efecto indirecto, ya sea porque tienen niveles altos de hábitos y conocimientos de base sobre el paludismo, o porque existe un efecto de contaminación de la intervención sobre los no intervenidos. En el primer caso, el hecho de vivir en la comunidad favorece el conocimiento de los tratamientos, el uso de toldillos y el permiso para la fumigación. Otros estudios han descrito el alto grado de conocimiento de la malaria en comunidades expuestas a la enfermedad durante 
largo tiempo o en zonas de alto riesgo $(8,21,22)$. Por ejemplo, entre los pobladores del Pacífico hay una alta proporción que se automedica (23), ya que los medicamentos se venden sin fórmula en las farmacias, lo que facilita su utilización y explica el conocimiento que se tiene de ellos. La alta proporción de uso del toldillo puede deberse a otras razones que no necesariamente se relacionan con el conocimiento de la prevención de la malaria (24). Los pobladores usan el toldillo para evitar el contacto con otros vectores, o el contacto con murciélagos o ratas (23). Esto explicaría la ausencia de efectos directos en esta práctica encontrada por nuestra evaluación.

En el segundo caso, los efectos indirectos encontrados pueden deberse a contaminación. Para averiguarlo, comparamos los datos de este estudio con otro de conocimientos y prácticas llevado a cabo en la misma zona en 1993. En el cuadro 4 se observa cómo en esta oportunidad se registraron más conocimientos sobre la malaria y mayores porcentajes de comportamientos preventivos y sintomáticos entre los encuestados que en el estudio de 1995 (13). Aunque el diseño y el método de muestreo fueron diferentes en los dos estudios, la similitud de las características generales de los entrevistados hace comparables nuestros datos con los del año 1993 (13). El aumento observado en dos años sugiere que parte del efecto encontrado en los "posiblemente no contacto" puede deberse al contacto indirecto con la intervención.

De todas maneras, si eliminamos este efecto indirecto, los resultados sugieren que educar a las personas en malaria o participar con ellas en procesos comunitarios facilita la adquisición de conocimientos y mejores prácticas, como lo demuestra el efecto total observado en esta evaluación. Igualmente, un efecto total se encontró para las prácticas del uso del toldillo y la fumigación, es decir que las personas intervenidas comparadas con aquellas que no tuvieron posibilidad de recibir nunca la intervención utilizaban más los toldillos, independientemente de las diferencias en el estado socioeconómico.

Aunque no se encontró efecto de la intervención en la frecuencia de la malaria en los 15 días anteriores, el análisis de tendencia de la malaria sí mostró una disminución en el número de casos en las comunas intervenidas. La baja frecuencia de reporte de la enfermedad en los 15 días previos puede explicar el hallazgo negativo. En la intervención aplicada en áreas rurales, cuando se preguntó por la frecuencia en los seis meses anteriores, sí se encontraron efectos directos (Alvarado BE, Gómez E, Serra M, Carvajal R, Carrasquilla G. Evaluación de una estrategia educativa en malaria aplicada en localidades rurales del Pacifico colombiano. Observaciones no publicadas).

\section{Limitaciones}

El diseño del estudio limita el alcance de algunas de estas conclusiones. Dado que la asignación de la intervención no fue al azar, los grupos de contacto y no contacto con la intervención pudieron ser heterogéneos en cuanto a factores relacionados tanto con la intervención como con el efecto buscado. En realidad, se encontró que las condiciones socioeconómicas y la escolaridad diferían en los tres grupos de contacto propuestos. La diferencia en la escolaridad puede mostrar cómo el programa buscó capacitar a personas más educadas, pensando que éstas podrían tener mayor capacidad de multiplicación y entender mejor el material educativo. De hecho, para el manejo del material educativo, saber leer es un requisito en la capacitación de los facilitadores. Sin embargo, el material educativo tiene herramientas que podían ser aplicadas por personas que no leen, como las láminas, los casetes y el video, y sobre las cuales se puede hacer énfasis en futuras intervenciones para alcanzar una mayor cobertura en las poblaciones.

Igualmente, las condiciones económicas de los "posiblemente no contacto" podrían explicar el menor uso de toldillos; en efecto, dichas personas relataban la falta de dinero como razón para no usarlos. Las asociaciones encontradas fueron ajustadas por variables de confusión, aunque no se puede descartar un efecto de confusión residual.

También es posible que el efecto limitado de la intervención sobre algunas prácticas y sobre la frecuencia individual de la malaria se deba a la baja cobertura del programa (especialmente en 
las comunas 10 y 11), sin descartar que para la definición de la exposición no se tomaron en consideración la intensidad y la calidad de la educación brindada por los facilitadores.

Es posible que un sesgo de historia (10) afecte los resultados. Por ejemplo, que otras intervenciones expliquen estos hallazgos y no la intervención aquí evaluada. Dado que las únicas actividades generadas en estos barrios fueron la fumigación y el control de criaderos terrestres encontrados por medio de la vigilancia (informe personal del director del programa de enfermedades tropicales en Buenaventura), esta disminución en el número de casos pudo deberse a las actividades de la intervención. Un diseño de estudio previo y posterior a la intervención hubiera sido ideal para evaluarla. Sin embargo, no se realizó un estudio previo a la evaluación en las comunidades intervenidas.

En conclusión, la educación sobre la malaria en las poblaciones facilita el cambio de comportamientos, lo cual puede repercutir en la frecuencia de casos. Los resultados de esta evaluación muestran la importancia de orientar las nuevas acciones hacia la comprensión del proceso visto desde la óptica de los facilitadores con el fin de mejorar el alcance del programa e incidir más en la percepción y en las conductas individuales de las personas. Además, el programa debe crear mecanismos para que la calidad y la cantidad del contacto con los agentes educativos se incremente y mejore. De igual manera, es importante evaluar el estado de la red de microscopía comunitaria y sus implicaciones en la detección y atención temprana de la malaria, pues dos estudios en la misma zona han evidenciado la baja utilización de este servicio.

\section{Agradecimientos}

Los autores agradecen la colaboración prestada por el educador social Luis Rubén Neira y el personal encuestador en el desarrollo del estudio.

\section{Conflictos de interés}

Este estudio no presenta ningún conflicto de intereses, ya que tres de los investigadores no participaron en la creación e implementación de la estrategia.

\section{Financiación}

Proyecto financiado por Fundación Antonio Restrepo Barco y Fundación FES Social.

\section{Referencias}

1. Najera JA. Global analysis of malaria situation to the development of Primary Health Care. Geneva; World Health Organization; 1986.

2. Najera JA. A suggested approach to malaria control and to the methodology applicable in different epidemiological situations based on experience in the Americas. Bull Pan Am Health Organ 1979;13:223-34.

3. Salcedo JM, Camargo EP, Krieger H, Silva LH, Camargo LM. Malaria control in an agro-industrial settlement of Rondonia (Western Amazon region, Brazil). Mem Inst Oswaldo Cruz 2000;95:139-45.

4. Nkuo Akenji TK, Ntonifor NN, Ching JK, Kimbi HK, Ndamukong KN, Anong DN, et al. Evaluating a malaria intervention strategy using knowledge, practices and coverage surveys in rural Bolifamba, southwest Cameroon. Trans R Soc Trop Med Hyg 2005;99:32532.

5. Kroeger A, Mancheno M, Alarcon J, Pesse K. Insecticide-impregnated bed nets for malaria control: varying experiences from Ecuador, Colombia, and Peru concerning acceptability and effectiveness. Am J Trop Med Hyg 1995;53:313-23.

6. Agudelo $\mathbf{C}$, Valero $\mathbf{M}$. Hacia un programa innovador del control de la malaria en el contexto del sistema general de seguridad social en salud en Colombia. Rev Salud Pública (Bogotá) 2004;6(Suppl.1):1-37.

7. Kroeger A, Avinna A, Ordoñez-Gonzalez J, Escandon C. Community cooperatives and insecticidetreated materials for malaria control: a new experience in Latin America. Malar J 2002;1:15.

8. Rojas W, Botero S, Garcia HI. An integrated malaria control program with community participation on the Pacific Coast of Colombia. Cad Saude Publica 2001;17 (Suppl.):103-13.

9. Carrasquilla $G$, Banguero $M$, Sanchez $P$, Carvajal F, Barker RH Jr, Gervais GW, et al. Epidemiologic tools for malaria surveillance in an urban setting of low endemicity along the Colombian Pacific coast. Am J Trop Med Hyg 2000;62:132-7.

10. Contandriopoulos AP, Champagne P, Potvin LD, Denis JL, Boyle P. Savoir preparer une recherche. Montreal: Les Presses de l'Universite de Montreal; 2005.

11. Carrasquilla G. An ecosystem approach to malaria control in an urban setting. Cad. Saúde Pública 2001;17 (Suppl.):171-9.

12. EPI-INFO. Word processing database and statistics system for epidemiology on microcomputers. In. 6.0 ed. Atlanta: CDC; 1996. 
13. Méndez F, Carrasquilla G. Epidemiología de la malaria en el área urbana de Buenaventura: análisis de la ocurrencia en el periodo 1987-1993. Colomb Med 1995;26:77-85.

14. Halloran ME, Haber M, Longini IM, Struchiner CJ. Direct and indirect effects in vaccine efficacy and effectiveness. Am J Epidemiol 1991;133:323-31.

15. STATA Corportation. STATA release 3.1. College Station Texas: Stata Corporation; 1997.

16. Tarimo E, Webster EG. Primary health care concepts and challenges in a changing world: Alma Ata revisited. Geneve: World Health Organization; 1993.

17. Chilaka MA. Ascribing quantitative value to community participation: a case study of the Roll Back Malaria (RBM) initiative in five African countries. Public Health 2005;119:987-94.

18. Sánchez $\mathbf{P}$. Uso de servicios de salud en la comuna 12 de Buenaventura: análisis de la prevalencia en un periodo de 1995 y sus factores determinantes. Cali: Universidad del Valle; 1997.

19. Cropley L. The effect of health education interventions on child malaria treatment seeking practices among mothers in rural refugee villages in Belize, Central America. Health Promot Int 2004;19:445-52.
20. Osorio LE, Giraldo LE, Grajales LF, Arriaga AL, Andrade AL, Ruebush TK 2nd, et al. Assessment of therapeutic response of Plasmodium falciparum to chloroquine and sulfadoxine-pyrimethamine in an area of low transmission in Colombia. Am J Trop Med Hyg 1999;61:968-72.

21. Kroeger A, Meyer R, Mancheno M, Gonzalez M Health education for community-based malaria control: an intervention study in Ecuador, Colombia and Nicaragua. Trop Med Int Health 1996;1:836-46.

22. Pineda F, Agudelo CA. Percepciones, actitudes y prácticas en malaria en el Amazonas colombiano. Rev Salud Publica (Bogota) 2005;7:339-48.

23. Mendez F. Epidemiología de la malaria en el área urbana de Buenaventura: análisis de ocurrencia en el periodo 1987-1993 y factores asociados con la prevalencia de infección en la comuna 12. Cali: Universidad del Valle; 1994.

24. Rodríguez AD, Penilla RP, Rodriguez M, Hemingway J, Betanzos AF, Hernandez JE. Knowledge and beliefs about malaria transmission and practices for vector control in southern Mexico. Salud Publica Mex 2003;45:110-6. 